\title{
Dynamic fragmentation of ceramics, signature of defects and scaling of fragment sizes
}

\author{
S. Levy, J.F. Molinari* \\ L.S.M.S - I.I.C - E.N.A.C, Ecole Polytechnique Fédérale de Lausanne, 1015 Lausanne, Switzerland
}

\section{A R T I C L E I N F O}

Article history:

Received 1 May 2009

Received in revised form

9 July 2009

Accepted 29 September 2009

\section{Keywords:}

Dynamic fragmentation

Expanding ring

Statistical distribution of defects

Average fragment size

Finite element simulations

\begin{abstract}
A B S T R A C T
Defects play a critical role in the dynamic fragmentation process of structural ceramics. Cracks initiate at seemingly random locations, propagate and coalesce to form fragments. The process is accompanied by stress release waves, whose influence is difficult to account for without numerical analysis. In this paper, we use a finite-element program with a cohesive fracture capability, to relate a defect distribution contained in a material with the resulting number of fragments. We show how the distribution tail, e.g. the number of large defects, and the rate at which cracks can be initiated at these sites have a critical influence on the generation of stress release waves and thus on the fragmentation process. Our numerical calculations yield a new factor, which we label communication factor, that we use to normalize the average fragment size and to define a new scaling function of material properties, defect statistics and loading rate.
\end{abstract}

(c) 2009 Elsevier Ltd. All rights reserved.

\section{Introduction}

Fragmentation, e.g. the breakage of a structure into smaller pieces, is a widespread phenomenon in nature and in engineering applications. It ranges from astrophysical scales such as in the creation of a galaxy (Ryan, 2000), to nanoscales e.g. fragmentation of DNA (Holian and Grady, 1988). In the intermediate range, fragmentation underlies many damage mechanisms, including ballistic impact, blast protection, explosive drilling in oil shales, debris after impact of satellites, car crashes, kidney stones fragmentation, and soil desiccation. During a dynamic and brittle fragmentation event, microcracks originate at seemingly random sites, open, propagate at large speeds, and eventually coalesce with one another to generate fragments. During their opening, microcracks release unloading waves that propagate around and protect the encompassed areas. Since each damaged site releases its own stress waves, a chaotic network of interactions emerges. Despite its complexity, fragmentation underlies so many applications that it has continuously appealed to the interest of the scientific community.

A range of phenomenological, theoretical and numerical models have been pursued during the last decades. Due to its violent nature, fragmentation was first described through its resulting state, making the observation of fragment sizes, shapes and velocities of considerable interest (Grady, 2009). Rosin and Rammler (1933) defined the most frequently quoted fragment size distribution of the thirties. Later, their empirical exponential law was confronted to the Mott-Linfoot's (1943) power law distribution. Besides its famous experiments, Mott (1943) opened the theoretical field of modeling fragmentation by studying the effect of wave propagation in a rapidly expanding ring. Since the eighties, numerous other descriptions have arisen. Based on energy balance principles, Grady's (1982) high strain rate theory predicts the

\footnotetext{
* Corresponding author.

E-mail address: jean-francois.molinari@epfl.ch (J.F. Molinari).
} 
average fragment size without taking account for wave interactions. Fragments are generated independently so that the local kinetic energy is fully converted into fracture energy. Glenn and Chudnovsky (1986) enriched Grady's model by adding the contribution of the elastic potential energy, which dominates at low loading rates. Due to their simplicity, these energy models constitute a reference frame for most engineering and research applications. However, they do not attempt to describe the evolution of the residual damage. Entropy models (Englman et al., 1987) couple continuum mechanics and thermodynamics principles, by including energy conservation and entropy production. Since they are based on empirical laws, these models are still debated. Nonetheless, all these theoretical frameworks do not account accurately for wave reflections and interactions. A variety of numerical methods have been developed to include them. For instance, Shenoy and Kim (2003), Drugan (2001), Zhou et al. (2006a) used the method of characteristics, but they were limited to onedimensional problems. The finite element method (Hughes, 2000), and the discrete element method handle more complex geometries. Coupled to the cohesive methodology, they offer an efficient compromise between accuracy and complexity. Maiti et al. (2005), Zhou et al. (2006b), Raghupathy et al. (2006), and Espinosa et al. (1998) used the finite element framework to simulate fragmentation. Wittel et al. (2005) presented a numerical study of shell fragmentation, based on the discrete element method. Even though their results seem hardly synthetizable into a simple analytical form, numerical simulations are able to represent accurately the evolution of the physical process.

Nevertheless, most of the prior theories consider fragmentation as a macroscopic process, without including the effect of the microstructure. In brittle fragmentation, fracture sites are usually structural flaws. A defect is activated when the stress is locally high enough to destroy the cohesion of the material. In quasi-statics, the weakest link theory applies (Weibull, 1939): the weakest flaws initiate and slowly lead to failure without crack interactions. In dynamics, numerous flaws are activated and interact through a complex network of stress waves. Each damaged defect opens and generates a stress release wave that protects the encompassed neighboring regions. When many defects open, stress waves are generated simultaneously, propagate, and interact. The behavior is then highly non-linear. Accounting for this wave effect and the density of activated defects, Hild et al. (2003) derived the tensile strength-rate corresponding to the transition between the quasi-static and the dynamic regimes. Recently, Paliwal and Ramesh (2008) developed a model for compressive fracture of heterogeneous materials that includes these interactions. Kraft et al. (2008) described numerically how grain boundary defects influence compressive fracture. However, relating microstructural defects and fragmentation remains an open issue, and is the central object of the present study.

Our objective is to extend the work of Zhou et al. (2006b) by including defect distributions. We wish to analyze how defects influence the average fragment size in an idealized system, consisting of a ceramic ring under uniform expansion. The ring material is initially elastic, and is controlled by a cohesive failure as soon as the stress field locally exceeds the strength of local flaws. The adopted numerical framework is the finite element method (FEM). The underlying question will be whether or not, despite the statistical complexity, our results can be synthesized in a simple normalized form, in which fragment statistics are made explicitly dependent on material properties, flaw population, and loading parameters. The paper is structured as follows. First, we describe the expanding ring test, which is chosen both for its simplicity and for its ability to involve only crack initiations (the effect of crack propagation is not analyzed in this paper). Then, we propose a method to define statistically a distribution of randomly spaced flaws. In the following sections, we first verify the numerical convergence of our results. Then, we discuss the influence of both the population of defects (density and representative distribution). A full section is devoted to building a theoretical model which aims at describing the communication between microcracks sites. Finally, we propose new scaling parameters for heterogeneous fragmentation.

\section{The numerical expanding ring test}

\subsection{Description of the test}

We study the fragmentation of a ring whose motion is imparted by some radial impulse or strain rate (Fig. 1):

$$
\dot{\varepsilon}=\frac{\text { radial velocity }}{\text { ring radius }}
$$

During the process, the input energy is converted into fracture energy, and into elastic and kinetic energies (due to the propagation of stress waves and to the global motion). In quasi-statics, the elastic energy dominates and the weakest link theory applies. On the contrary, the dynamic regime is governed by kinetic effects, which are arguably more challenging to understand. Therefore, our applications concentrate on strain rates ranging from $10^{3}$ to $10^{6} \mathrm{~s}^{-1}$. Moreover, we have chosen this test for its simplicity. The radial and periodic geometry limits boundary effects and contacts between fragments. The small thickness of the ring restricts the fragmentation process to crack initiation. Since crack propagation makes the physical understanding more complex, focusing on initiation rather than propagation is clearly an advantage to complete our objectives.

Before fracture, the behavior is linear elastic and controlled by a finite element simulation (Section 2.2). When the weakest defects are initiated, cohesive elements are inserted and stress release waves begin their propagation and interactions. Depending on the local stress, cracks may nucleate, grow, or close (Section 2.3). As soon as one link is completely broken, the expansion of the ring is not constrained anymore: inertial effects govern the evolution of the 


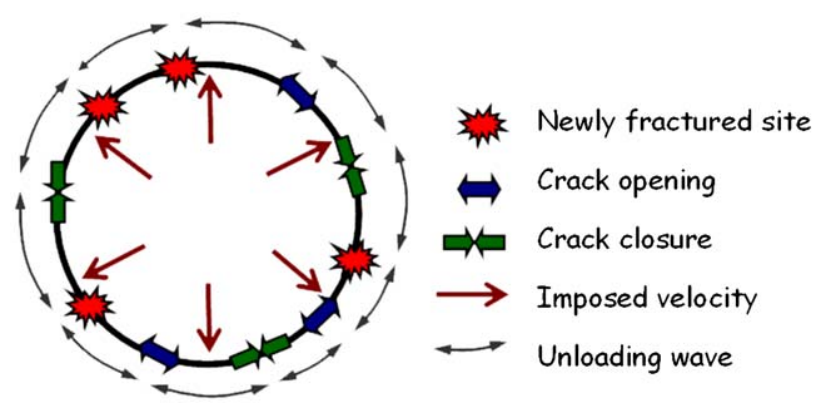

Fig. 1. Schematic of the expanding ring test showing the development of a complex network of stress wave interactions.

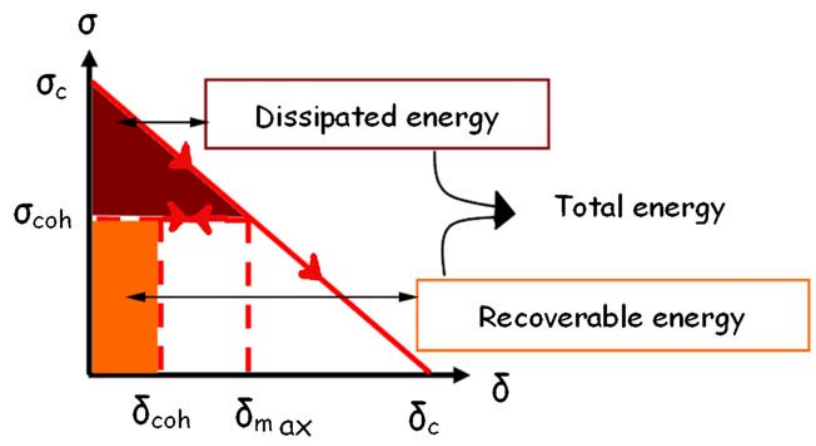

Fig. 2. Schematic of the linear cohesive law and the associated energies.

fragmentation. While the ring continues expanding, cracks keep evolving and interacting until the fragmentation process is stabilized.

\subsection{Finite element discretization}

The ring expansion is computed by using a finite element code. The mesh represents a two-dimensional ring of circumferential length $L_{\text {ring }}=50 \mathrm{~mm}$, with one element in the thickness. Elements are six-node triangles. Their number depends on the applied strain rate, and is sufficiently large to obtain converged numerical results (Raghupathy et al., 2006) (Section 3.1). The material is a fictitious ceramic with volumetric mass $\rho=2750 \mathrm{~kg} \mathrm{~m}^{-3}$, Young's modulus $E=275 \mathrm{GPa}$, and Poisson ratio $v=0.3$, that behaves linearly before fracture. The wave speed is $c=\sqrt{E / \rho}=10000 \mathrm{~m} \mathrm{~s}^{-1}$.

The discretization in space is commonly based on the principle of virtual work:

$$
\mathbf{M} \ddot{\mathbf{x}}+\mathbf{R}^{\text {int }}(\mathbf{x})=\mathbf{R}^{\text {ext }}(x)
$$

where $\mathbf{R}^{\text {int }}$ and $\mathbf{R}^{\text {ext }}$ are the internal and external force arrays, $\mathbf{M}$ is the lumped mass matrix and $\mathbf{x}$ is the nodal coordinate array.

The discretization in time is based on an explicit central difference scheme (Hughes, 2000) which limits the time scale. The Newmark theory leads to the definition of a stable time scale $\Delta t_{\text {stable. }}$. In all the simulations, we use $\Delta t$ such that

$$
\Delta t=S \Delta t_{\text {stable }}=\frac{S}{c} \min _{e \in \text { mesh }}\left(h_{e}\right)
$$

where $S$ is a security coefficient (typically equal to 0.1 ), and $h_{e}$ is the characteristic dimension of the element $e$.

\subsection{Cohesive element methodology}

The cohesive element methodology drives the failure behavior. We follow the derivations of Camacho and Ortiz (1996) for an initially rigid irreversible law. A threshold criterion controls the damage onset: a defect is activated as soon as the local stress $\sigma$ exceeds its cohesive strength $\sigma_{c}$. When the cohesive element is inserted, the edges associated to the defect can open. The damage behavior follows the linear decreasing cohesive law which relates the local stress $\sigma_{\text {coh }}$ and the opening displacement $\delta_{c o h}$ (Fig. 2). As long as the nodes do not contact, the cohesive law takes the form

$$
\frac{\sigma_{c o h}}{\sigma_{c}}=1-\frac{\delta_{c o h}}{\delta_{c}} \quad \text { for } \dot{\delta}_{c o h}>0, \delta_{c o h}=\delta_{\max } \text { and } D<1
$$




$$
\frac{\sigma_{c o h}}{\sigma_{c}}=1-\frac{\delta_{\max }}{\delta_{c}} \text { for } \delta_{c o h}<\delta_{\max } \text { and } D<1
$$

The first and the second equations, respectively, govern the element opening and the element closing. The local damage $D$ is directly related to the maximum opening. It is comprised between 0 (initiation of the damage) and 1 (the fracture site is fully broken):

$$
D=\min \left(\frac{\delta_{\max }}{\delta_{c}}, 1\right)
$$

Note that the unloading slope is unconventional and necessary because the problem is one-dimensional (Zhou et al., 2005). The interpenetration between elements is controlled by adding of a repulsive force, proportional to the interpenetration, such that the contact nodes are artificially separated. The energy associated to the defect opening is divided into a dissipated part $E_{\text {diss }}$ and a recoverable part $E_{\text {rec }}$ (Fig. 2). When the cohesive element is completely broken, it has released the toughness $G_{c}$ :

$$
G_{c}=\frac{\sigma_{c} \delta_{c}}{2}
$$

When it is partially opened, it has dissipated $E_{\text {diss }}$ and retains $E_{\text {rec }}$ :

$$
\begin{aligned}
& E_{\text {diss }}=D^{2} G_{c} \\
& E_{\text {rec }}=\sigma_{c o h} \delta_{c o h}=2(1-D) \frac{\delta_{c o h}}{\delta_{c}} G_{c}
\end{aligned}
$$

When the crack is on the opening path, $\delta_{\text {coh }}=\delta_{\max }$ and the recoverable energy is

$$
E_{\text {rec }}=2(1-D) D G_{C}
$$

\subsection{Modeling the initial distribution of defects}

Every material is inherently imperfect; defects are distributed among its volume. Each defect is naturally associated to a failure strength, which we refer to as its cohesive strength $\sigma_{c}$. It designates the stress activation threshold required to initiate fracture, and its value depends on its shape, size and orientation. A defect with a low failure strength is weak, while a defect with a large failure strength has a low probability of failure. Since each defect is affiliated to a value of $\sigma_{c}$, statistical heterogeneity is introduced with a distribution of $\sigma_{c}$. A population of defects is thus characterized by:

1. the number of defects $N_{\text {def }}$, or the defect density $L_{\text {ring }} / N_{d e f}$;

2. the type of the distribution of defects (normal, uniform, etc.);

3. the mean $\mu$ and the standard deviation $v$ of the distribution.

Choosing an adequate distribution is not obvious. We have focused on the uniform distribution which is not realistic, but whose simple shape helps build a physical understanding. Then, we have compared our results to Gaussian and Weibull distributions. These choices were made with respect to the weakest link theory and to extreme value statistics (Leadbetter et al., 1983). The cumulative density function of the Weibull distribution is defined by

$$
F\left(\sigma_{c}\right)=1-e^{-\left(\left(\sigma_{c}-\sigma_{c, \min }\right) / \lambda\right)^{m}}
$$

where $\lambda$ is the scale parameter, $m$ is the shape parameter or Weibull modulus, and $\sigma_{c, \min }$ is the minimum value of the cohesive strengths.

\begin{tabular}{|c|c|c|c|c|c|c|c|}
\hline Distribution name & Gauss 2 & Weibull 2-1 & Weibull 2-2 & Weibull 10 & Weibull 20 & Uniform 1 & Uniform 20 \\
\hline Mean $\mu$ & 345 & 345 & 327 & 347 & 348 & 345 & 348 \\
\hline Standard deviation $v$ & 23.2 & 23.2 & 95.7 & 5.7 & 3.0 & 140.0 & 3.0 \\
\hline Minimum value $\sigma_{c, \min }$ & $\mathrm{x}$ & 300 & 150 & 300 & 300 & 102.5 & 342.8 \\
\hline Weibull modulus $m$ & $\mathrm{x}$ & 2 & 2 & 10 & 20 & $\mathrm{x}$ & $\mathrm{x}$ \\
\hline Scale parameter $\lambda$ & $\mathrm{x}$ & 5 & 200 & 5 & 5 & $\mathrm{x}$ & $\mathrm{x}$ \\
\hline
\end{tabular}

In the present paper, several distributions of defects are thus tested. Table 1 details their mathematical characteristics. Fig. 3 illustrates some of the probability density functions (p.d.f.). The chosen Weibull distributions, which are considered by the community to be realistic mathematical models for defects, cover a wide range of standard deviations and Weibull moduli.

Table 1

Theoretical characteristics of the defect distributions.

Stresses are expressed in MPa. 


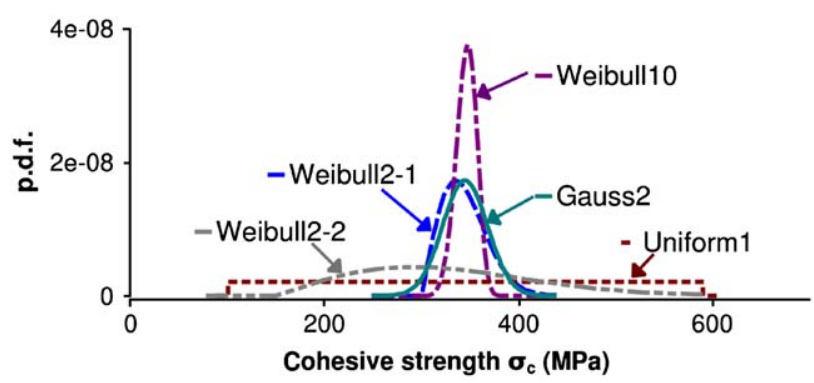

Fig. 3. Some realizations of cohesive strengths (p.d.f.).

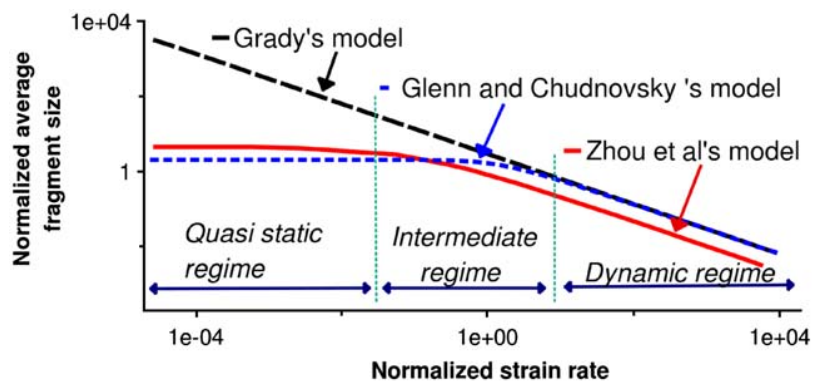

Fig. 4. Reference models for homogeneous fragmentation.

\subsection{Brief review of prior theories}

During the past decades, this simple expanding ring test has appealed to so many researchers that an extensive review of the prior work is inadequate. Therefore, we only describe the theory derived by Grady (1982), extended by Glenn and Chudnovsky (1986), and the recent numerical results of Zhou et al. (2006c). They all propose a law relating the number of fragments and the strain rate in the case of the fragmentation of a homogeneous material. The fracture energy $G_{c}$ and the cohesive strength $\sigma_{c}$ are thus independent from the spatial coordinates, and each fracture site dissipates the same amount of energy $G_{c}$.

Grady, and Glenn and Chudnovsky had recourse to an energy argument to derive the expression of the average fragment size. Employing the cohesive methodology, Zhou et al. (2006c) detailed the effect of wave interactions and normalized their results, making implicit the dependence on material parameters in a simple equation. The normalization involves three relevant parameters: $s_{0}, t_{0}$ and $\dot{\varepsilon}_{0}$. The characteristic length $s_{0}$ represents the size of a fragment in quasi-statics, when the applied potential energy is fully converted into fracture energy. This length $s_{0}$ is directly related to the characteristic time $t_{0}$ defined by Camacho and Ortiz (1996). This time $t_{0}$ expresses the time needed by the waves, released by a cohesive element, to fully encompass $s_{0}$. Another relevant parameter is the characteristic strain rate $\dot{\varepsilon}_{0}$ defined by Drugan (2001). A possible interpretation relates $\dot{\varepsilon}_{0}$ and $t_{0}: t_{0}$ is the time needed by the cohesive element to fully open when submitted to $\dot{\varepsilon}_{0}$. The analytical expressions of these three representative parameters are

$$
\begin{gathered}
t_{0}=\frac{E G_{c}}{\sigma_{c}^{2} c} \\
s_{0}=c t_{0} \\
\dot{\varepsilon}_{0}=\frac{\sigma_{c}}{E t_{0}}
\end{gathered}
$$

Fig. 4 represents Grady's, Glenn and Chudnovsky's, and Zhou et al.'s models with normalized axes. The normalized strain rate and the normalized average fragment size are defined in Zhou et al. (2006c) by

$$
\bar{s}=\frac{s}{s_{0}} \quad \text { and } \quad \overline{\dot{\varepsilon}}=\frac{\dot{\varepsilon}}{\dot{\varepsilon}_{0}}
$$

Using this notation, our reference equations are the two theoretical models based on energy conservation (Eqs. (16) and (17)) and the numerical model which includes wave propagation and dynamic effects (Eq. (18)):

$$
\text { Grady's model : } \quad \bar{s}_{G}=\left(\frac{24}{\overline{\dot{\varepsilon}}^{2}}\right)^{1 / 3}
$$


Glenn and Chudnovsky's model : $\quad \bar{S}_{G C}=\frac{4}{\dot{\dot{\varepsilon}}} \sinh \left(\frac{1}{3} \sinh ^{-1}\left(\frac{3}{2} \overline{\dot{\varepsilon}}\right)\right)$

Zhou et al.'s model : $\quad \bar{s}_{Z M R}=\frac{4.5}{1+4.5 \overline{\dot{\varepsilon}}^{2 / 3}}$

Nevertheless, these normalizations only address the homogeneous case. In this paper, since we are dealing with a distribution of fracture toughnesses and a distribution of cohesive strengths, the parameters $G_{c}$ and $\sigma_{c}$ are not clearly defined anymore. In order to simplify the problem, we have constrained the parameter $t_{0}$ to be identical for all cohesive elements. This implies that all the cohesive elements have the same intrinsic opening time. This choice facilitates the understanding of the phenomenon without a too severe loss of generality. We still account for a distribution of $\sigma_{c}$ and a distribution of $G_{c}$; the only difference is that they are linked to one another by

$$
\sigma_{c}=\sqrt{\frac{E}{s_{0}} G_{c}}
$$

To determine $s_{0}$, we have chosen the reference values $\sigma_{c 0}=300 \mathrm{MPa}$ and $G_{c 0}=100 \mathrm{~N} \mathrm{~m}^{-1}$.

\section{Numerical convergence and defect density}

\subsection{Influence of the number of elements $N_{F E}$}

Our finite elements discretization is controlled by two independent parameters: the number of elements of the finite element computation $N_{F E}$, and the number of defects $N_{d e f}$. Contrary to the number of defects $N_{d e f}$ which is a physical parameter standing for the density of defects, the number of elements $N_{F E}$ is purely numerical. It determines the numerical convergence of the simulations. In this section, we focus on the effect of $N_{F E}$. The next section is dedicated to studying the effect of $N_{\text {def }}$.

Verifying the numerical convergence usually consists in setting the number of defects $N_{\text {def }}$ to given values and varying the number of elements $N_{F E}$. This convergence issue has already been addressed for homogeneous materials in Raghupathy et al. (2006), but, as far as we know, has not be dealt for heterogeneous materials. Fig. 5 underlines that, for a given number of defects $N_{\text {def }}$, the number of fragments $N_{\text {frag }}$ is a slightly decreasing function of the number of elements, as long as the mesh is fine enough. For instance, when $N_{d e f}=8000$, the number of fragments varies from 1485 ( $\left.N_{F E}=20000\right)$ to 1461 $\left(N_{F E}=35000\right)$. Each point corresponds to one simulation carried out at the strain rate $10^{6} \mathrm{~s}^{-1}$ for a Gaussian distribution with mean $\mu=345 \mathrm{MPa}$ and standard deviation $v=23.2 \mathrm{MPa}$ (Gauss 2 in Table 1 ).

As a result, we have set the number of elements $N_{F E}$ to 30000 , which guarantees the convergence for every strain rate lower than $10^{6} \mathrm{~s}^{-1}$. Besides, one should note that, when the number of elements $N_{F E}$ and the number of defects $N_{d e f}$ have close values, the numerical simulations are very unstable and generally lead to the full explosion of the ring, breaking all the cohesive elements. In general, it is advised to set

$$
N_{F E}>2.5 N_{d e f}
$$

Convergence can also be verified by varying the number of elements $N_{F E}$, while keeping the ratio $N_{\text {def }} / N_{F E}$ constant. Fig. 6 underscores that the convergence is guaranteed for the four tested ratios. The rate of convergence is lower for small ratios because the number of defects increases slower. As explained in Section 3.2, the converged number of fragments depends on the ratio because of defect density effect.

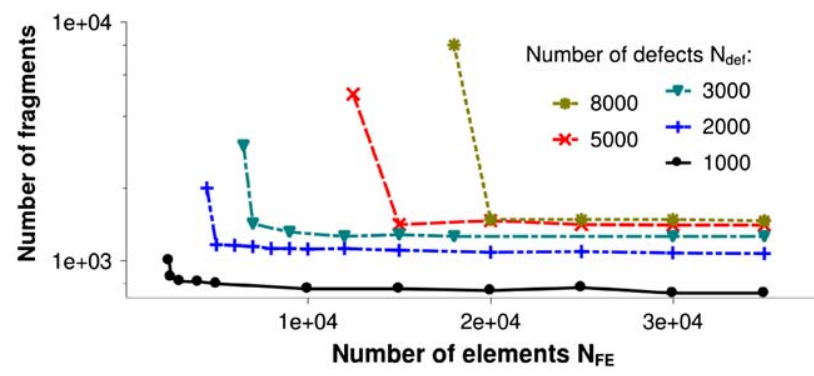

Fig. 5. Numerical convergence of the number of fragments $N_{\text {frag }}$ for a constant number of defects $N_{\text {def }}$. 


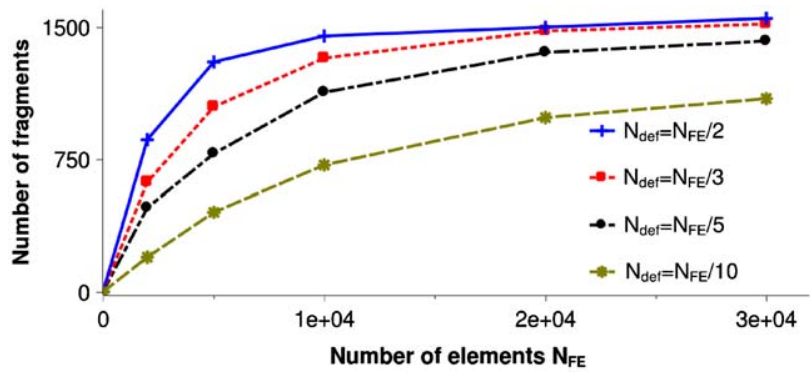

Fig. 6. Numerical convergence of the number of fragments $N_{\text {frag }}$ for a constant ratio $N_{\text {def }} / N_{F E}$.

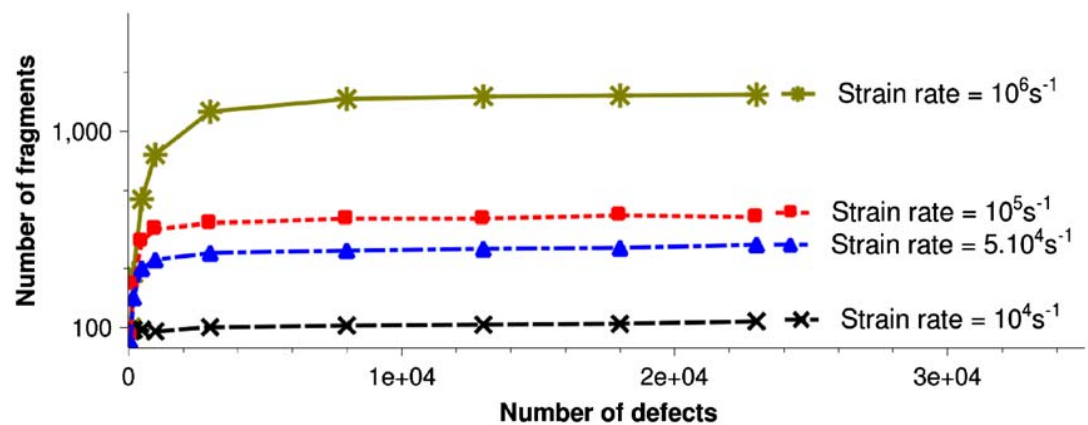

Fig. 7. Evolution of the number of fragments with the number of defects.

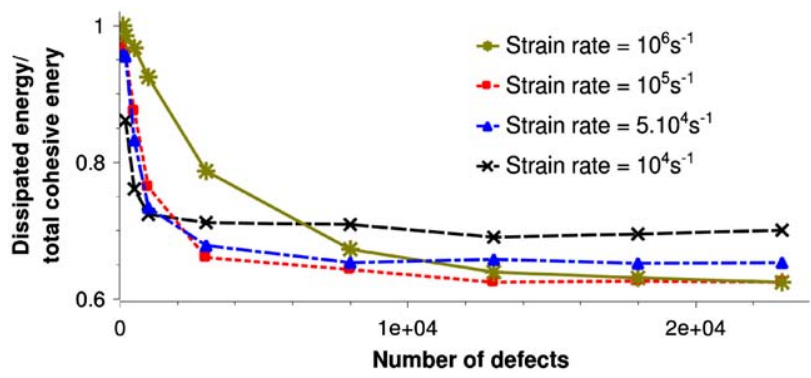

Fig. 8. Evolution of the rate of the dissipated cohesive energy and the total cohesive energy, with the number of fragments.

\subsection{Influence of the number of defects $N_{\text {def }}$}

\subsubsection{Proportion of the broken defects $N_{\text {def }}^{\text {bron }}$}

In this section, we look at the influence of the number of defects on the fragmentation process. We set the number of elements to 30000 , and the p.d.f. of the initial distribution to be Gaussian, with mean $\mu=345 \mathrm{MPa}$ and standard deviation $v=23.2 \mathrm{MPa}$ (Gauss 2 in Table 1 ). We let the number of defects $N_{\text {def }}$ vary, and we count the number of fragments $N_{\text {frag }}$. If there are few initial defects in the ring, they will all break. However, if we consider a higher defect density, only a part of the defects will break while the rest will get partially damaged or will remain fully intact.

Fig. 7 plots the number of fragments $N_{\text {frag }}$ versus the number of defects $N_{\text {def }}$. As there is only one element in the thickness, we can easily relate the number of fragments and the number of broken defects:

$$
N_{\text {frag }}=N_{\text {def }}^{\text {broken }}+1
$$

Fig. 8 represents the evolution of the ratio $E_{\text {diss }} /\left(E_{\text {diss }}+E_{\text {rec }}\right)$ with the number of defects. The total cohesive energy stands for the sum of dissipated and recoverable energies $\left(E_{\text {diss }}+E_{\text {rec }}\right)$. Obviously, the curves in Fig. 7 are monotonically increasing, while they are monotonically decreasing in Fig. 8. The proportion of the dissipated cohesive energy with respect to the total cohesive energy gets smaller as the number of defects, or number of potential dissipative links, increases. Three phases arise:

(i) All the defects break: $N_{\text {def }} \simeq N_{\text {def }}^{\text {broken }}$. The total cohesive energy takes mainly the form of dissipated energy, which means that there are only a few cohesive elements that store recoverable energy, or that only a few defects are partially damaged. Most of them are fully broken. 
(ii) Defects are either broken or damaged: $N_{\text {def }} \simeq N_{\text {def }}^{\text {bron }}+N_{\text {def }}^{\text {damaged }}$. The cohesive energy is divided into recoverable and dissipated energies.

(iii) Defects are either broken, damaged, or intact: $N_{\text {def }}=N_{\text {def }}^{\text {broken }}+N_{\text {def }}^{\text {damaged }}+N_{\text {def }}^{\text {intact }}$. Raising $N_{\text {def }}$ does not affect significantly $N_{d e f}^{b r o k e n}$ anymore. Fig. 8 underlines that the proportion of the recoverable and the dissipated energies is also stable. This steady value depends on the strain rate, which appears to be a decreasing function of the strain rate. For instance, when $\dot{\varepsilon}=10^{4} \mathrm{~s}^{-1}$, it is about 0.70 , while $\dot{\varepsilon}=10^{6} \mathrm{~s}^{-1}$ leads to a value of 0.62 . At a given strain rate, it guarantees that raising the number of defects in the ring has no effect neither on $N_{\text {def }}^{\text {broken }}$ nor on $N_{\text {def }}^{\text {damaged }}$.

The steady regime (iii) is a consequence of stress wave relaxation. Indeed, each damaged fracture site releases a wave that unloads partially the encompassed regions. The fragmentation process reaches its final state when the waves have sufficiently unloaded the body. As a result, with increasing number of defects, more sites possibly become damaged and release stress waves. This amounts to a faster fragmentation process. Since it cannot be infinitively short, the process characteristic time reaches a lower value, irrespective to the number of defects. Hence, in this regime, increasing the number of defects does not alter the final stage.

In practice, working on the steady phase (iii) requires to have enough defects on the ring. The transition value between phases (ii) and (iii) depends on the strain rate. In phase (iii), any rise in the number of initial defects will have no consequence in the results, in terms of number of fragments and cohesive energies. In the following sections, the number of defects is set such that we are certain to work in phase (iii), keeping in mind that the simulation duration increases with the number of initial defects.

\subsubsection{Representation in terms of average fragment size}

In order to compare our results to Grady's and Zhou et al.'s models, we have normalized the axes following Section 2.5, considering that the material is homogeneous. This hypothesis is evidently wrong, but is a priori necessary to compare results. The issue of normalization for heterogeneous materials is handled in Section 5. Fig. 9 represents the evolution of the normalized average fragment size versus the normalized strain rate, for different values of $N_{\text {def }}$.

We underline the influence of $N_{\text {def }}$ by computing the average slope of each plot, and compare it to Grady's model. Fig. 10 illustrates that it is a monotonically decreasing function with an asymptotic limit. For $N_{\text {def }}=100$, the slope is closed to zero which expresses that all the defects are broken (phase (i)). As $N_{\text {def }}$ increases, the slope decreases (phase (ii)) until it reaches the steady region for $N_{\text {def }} \geq 10000$ (phase (iii)). Although this asymptotic value is slightly different from Grady's, its minus two-thirds theoretical prediction appears to be a very good approximation for the present one-dimensional test.

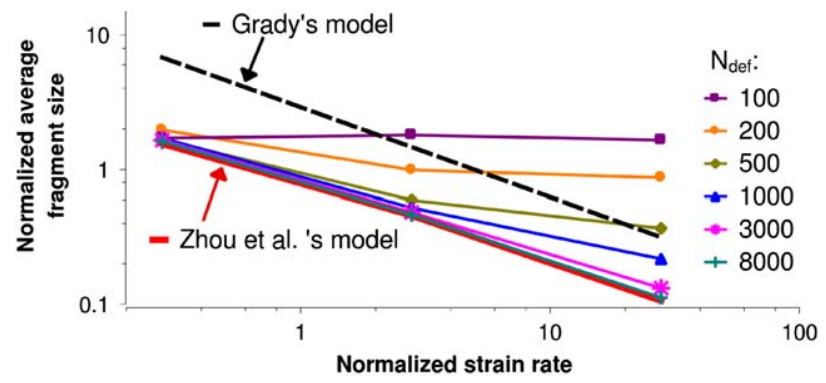

Fig. 9. Normalized representation of the average fragment size for several number of defects.

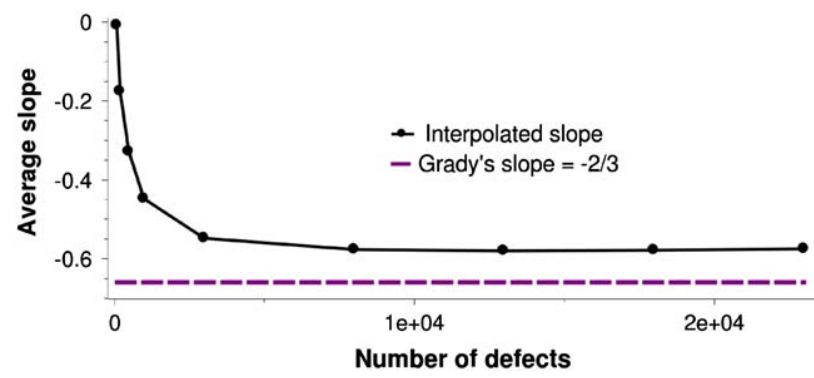

Fig. 10. Evolution of the average slope of the plots in Fig. 9 in the dynamic regime, with the number of defects. 


\section{Keys to understand communication between defects}

\subsection{Qualitative understanding}

Being able to predict how crack interactions affect fragmentation is challenging. In the present article, we take advantage from the simplicity of the expanding ring test to develop a comprehensive measure of crack interactions. When the ring fragments, some defects are initiated, they open and release stress waves that propagate and protect the encompassed regions. An opening defect interacts with its neighbors through these waves. If they propagate fast, the waves encompass a large region which may prevent numerous defects from getting damaged. Hild et al. (2003) went further by considering that once a defect is encompassed by a wave, it is definitively protected and cannot get damaged anymore. In this case, the communication between defects is then prevailing. We call it the extremely communicative process. On the contrary, if they propagate slowly, less defects are encompassed, and little interactions take place: the communication is inconsequential. This without communication approach was derived by Grady $(1982,1988)$ whose pioneering energy theory does not include any crack interactions. The next paragraphs are dedicated to compare these two extreme behaviors in order to quantify the degree of communication for any non-extreme case.

\subsubsection{Characterization of the communication extreme cases}

Without communication: The stress waves are propagating slowly, compared to the crack opening, and do not have time to transmit the information. Consequently, each fracture site behaves independently from the others. The time associated to the crack opening $t_{0}$ is much smaller than the characteristic wave propagation time $L_{\text {ring }} / c$. In the non-communicative fragmentation case, the weakest flaw first is initiated; then, since they do not interact, the second weakest flaw gets initiated, then the third. The process continues until all the fracture energy is dissipated. Only the weakest defects are damaged (Fig. 11). Instantaneous crack opening, extremely slow wave propagation, fragility, and the absence of communication are thus directly related. Moreover, when a weak defect breaks, it releases a small amount of energy which is proportional to its associated $\sigma_{c}$ (Eq. (19)). Dissipating totally the input strain energy will thus require the activation of numerous flaws. The non-communicative process may consequently generate "numerous" fragments.

With intense communications: Defects communicate intensively through stress waves that are propagating fast in comparison to the crack opening. The time associated to the crack opening $t_{0}$ is thus much larger than the characteristic wave propagating time $L_{\text {ring }} / c$. When the stress waves propagate fast enough, they encompass defects that are associated to any critical failure strength $\sigma_{c}$. Weakest and strong defects may either be protected or damaged, depending on whether or not they have been encompassed by a stress release wave (Fig. 11). Slow crack opening, fast wave propagation, ductility, and high communication rate are thus associated. Moreover, considering that a strong defect breakage releases a large amount of energy, this intensely communicative process may result in a "small" number of fragments.

Effective wave speed: Hence, the same opening time $t_{0}$ and the same wave speed $c$ can lead to two opposite behaviors. One possible way of taking this remark into account is to define an effective wave speed $c_{e f f}$. It measures the interactions between defects during any fragmentation process. The following paragraphs are dedicated to define and quantify it.

\subsubsection{Definition of relevant parameters}

Fig. 11 illustrates an initial probability density function $f^{\text {init }}$, the two extreme cases previously detailed, and an intermediate possibly real one (the hatched curves do not represent p.d.f. because their area is smaller than one). The hatched areas represent the proportion of the broken defects, and are related to the number of defects $N_{d e f}$ and the number of fragments $N_{\text {frag. }}$. We denote these areas by $\alpha$ :

$$
\alpha=\frac{N_{\text {frag }}}{N_{\text {def }}}
$$

Contrary to the weakest link which is always activated, the communication affects the average and the maximum failure strengths of the broken defects, respectively, denoted by $\mu^{\text {broken }}$ and $\sigma_{c, \max }^{\text {broken }}$. A small $\sigma_{c, \max }^{\text {broken }}$ expresses a low communication

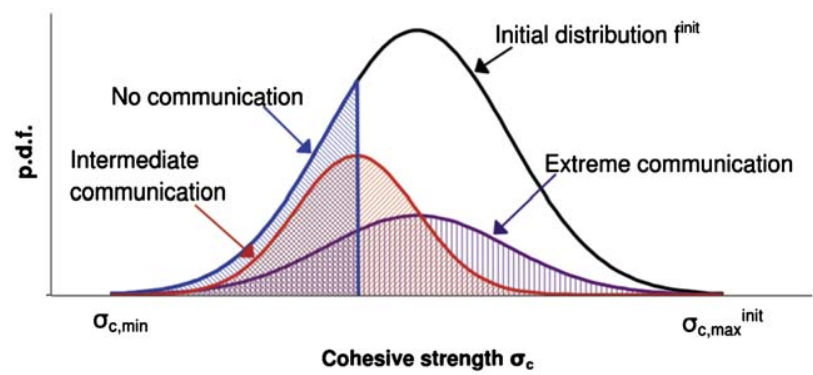

Fig. 11. Schematic of the broken defects, within the same initial distribution: the extremely communicative, the without communication and one possibly real cases. Note: $\sigma_{c, \min }=\sigma_{c, \text { min }}^{\text {init }}=\sigma_{c, \text { min }}^{\text {broken }}$ and $\sigma_{c, \text { max }}^{\text {init }} \neq \sigma_{c, \text { max }}^{\text {broken }}$. 
rate, while a large one underscores that interactions are prevailing. The two extreme cases follow therefore the properties:

Without communication process :

$$
\begin{aligned}
& \left\{\begin{array}{l}
\sigma_{c, \text { min }}^{\text {broken }}=\sigma_{c, \text { min }}^{\text {init }}=\sigma_{c, \text { min }} \\
\mu^{\text {broken }}<\mu^{\text {init }} \\
\sigma_{c, \text { max }}^{\text {broken }}<\sigma_{c, \text { max }}^{\text {init }} \\
f^{\text {broken }}= \begin{cases}\frac{f^{\text {init }}}{\alpha} & \text { if } \sigma_{c}<\sigma_{c, \text { max }}^{\text {broken }} \alpha=\int_{\sigma_{c, \text { min }}}^{\sigma_{\text {broen }}^{\text {bron }}} f^{\text {init }} \\
0 & \text { otherwise }\end{cases}
\end{array}\right. \\
& \text { Extremely communicative process : }\left\{\begin{array}{l}
\sigma_{c, \text { min }}^{\text {broken }}=\sigma_{c, \text { min }}^{\text {init }}=\sigma_{c, \text { min }} \\
\mu^{\text {broken }} \simeq \mu^{\text {init }} \\
\sigma_{c, \text { max }}^{\text {broken }} \simeq \sigma_{c, \text { max }}^{\text {init }} \\
f^{\text {broken }} \simeq f^{\text {init }}
\end{array}\right.
\end{aligned}
$$

In the following section, we will therefore focus on $\alpha, \sigma_{c, \min }, \mu^{\text {init }}$ and $\mu^{\text {broken }}$ by studying the evolution of the function $\Psi$ defined by

$$
\begin{aligned}
& \Psi:[0,1] \rightarrow[0,1] \\
& \alpha \rightarrow \frac{\mu^{\text {broken }}-\sigma_{c, \text { min }}}{\mu^{\text {init }}-\sigma_{c, \text { min }}}
\end{aligned}
$$

The variation of the parameter $\alpha$ can be achieved either by changing the number of initial defects $N_{d e f}$, or by changing the strain rate $\dot{\varepsilon}$ which affects the number of fragments $N_{\text {frag }}$. We have also studied the evolution of the maximum and the standard deviation of the broken distribution, and have drawn similar conclusions than the ones detailed in the following. Consequently, for reasons of conciseness, they will not be presented in the present paper.

\subsection{Quantitative measure of the communication between defects}

\subsubsection{Studied distributions}

We now vary the initial p.d.f. and compare the degree of communication they result in. For each initial p.d.f. $f^{\text {init }}$, we expect the numerical simulations to lead to a p.d.f. of the broken defects $f^{\text {broken }}$ with a communication rate between the two previously described extreme cases. The results obtained for three different initial p.d.f. $f^{\text {init }}$ are presented here (Fig. 3 ):

- A uniform distribution with a large standard deviation $v^{\text {init }}=140 \mathrm{MPa}$ and a mean $\mu^{\text {init }}=345 \mathrm{MPa}$. It corresponds to the case Uniform 1 in Table 1. The slope of the p.d.f. is zero everywhere, except at the minimum and the maximum values $\sigma_{c, \min }^{\text {init }}$ and $\sigma_{c, \max }^{\text {init }}$ where it is infinite. This high slope at $\sigma_{c, \text { min }}^{\text {init }}=102.5 \mathrm{MPa}$ should lead to the activation of many defects at the same instant, so that they may not have time to communicate. This large activation rate should thus result in a low communication rate.

- A Gaussian distribution with a standard deviation $v^{\text {init }}=23.2 \mathrm{MPa}$ and a mean $\mu^{\text {init }}=345 \mathrm{MPa}$. It corresponds to the case Gauss 2 in Table 1 . Since the slope at $\sigma_{c, \min }^{\text {init }} \simeq 245 \mathrm{MPa}$ is nearly zero, it tends to smooth the fragmentation process, and the interactions should have time to establish. This low activation rate of defects should thus result in a large communication rate.

- A Weibull distribution with a standard deviation $v^{\text {init }}=23.2 \mathrm{MPa}$ and a mean $\mu^{\text {init }}=345 \mathrm{MPa}$. It corresponds to the case Weibull 2-1 in Table 1 . This p.d.f. should lead to an intermediate behavior since the slope at $\sigma_{c, m i n}^{\text {init }}=300 \mathrm{MPa}$ is comprised between zero and infinity.

Besides, in order to justify more rigorously the influence of the slope of $f^{\text {init }}$ at $\sigma_{c, \text { min }}^{\text {init }}$, we have also tested the distributions represented in Fig. 12. Although they do not probably correspond to any real distribution of defects, these p.d.f. highlight the qualitative trend which relates the communication behavior to the rate of insertion of cohesive elements, and to the slope of $f^{\text {init }}$ at $\sigma_{c, \text { min }}^{\text {init }}$. We set $\sigma_{c, \text { min }}^{\text {init }}=307 \mathrm{MPa}, \sigma_{c, \text { max }}^{\text {init }}=381 \mathrm{MPa}$ and vary the intermediate $\sigma_{c, \text { intermediate }}^{\text {init }}$ such that

$$
\sigma_{c, \text { intermediate }}^{\text {init }}=\sigma_{c, \min }+\beta\left(\sigma_{c, \max }^{\text {init }}-\sigma_{c, \min }\right) \quad \text { where } \beta \in[0,1]
$$

\subsubsection{Definition and determination of the communication parameter}

Our main concern is the study of the monotonously increasing function $\Psi$ (Eq. (23)) with the aim at determining a relevant communication parameter. Evidently, $\Psi(0)=0$ and $\Psi(1)=1$. Fig. 13 underlines that, depending on the communication rate, several curves may link these two points. In the case of intense communication, we have shown that $\sigma_{c, \text { max }}^{\text {broken }} \simeq \sigma_{c, \text { max }}^{\text {init }}$, which corresponds to a Heaviside step function. Reversely, the case without communication links directly the 


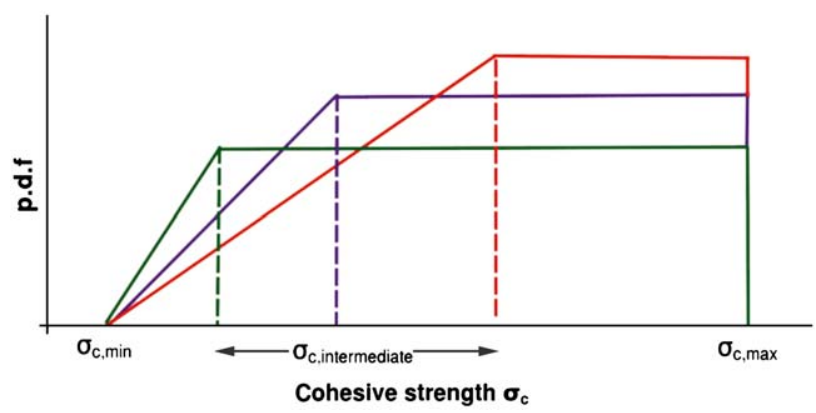

Fig. 12. Schematic of the initial distributions of defects generated to control the influence of the slope at $\sigma_{c, \text { min }}$ on the communication behavior.

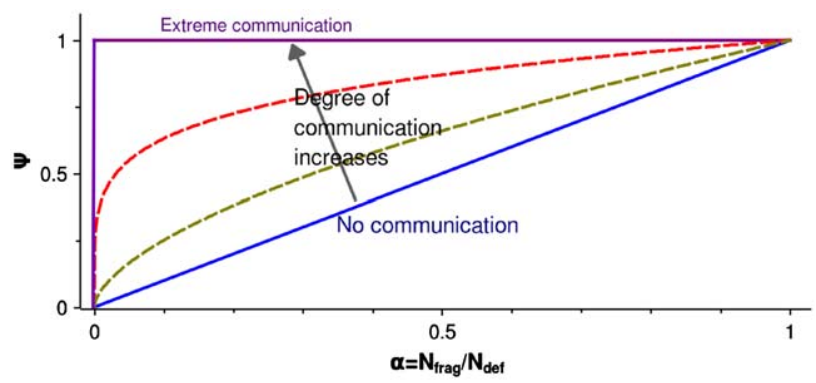

Fig. 13. Schematic of the theoretical effect of the communication rate on the function $\Psi$ for a uniform initial distribution of defects finit.

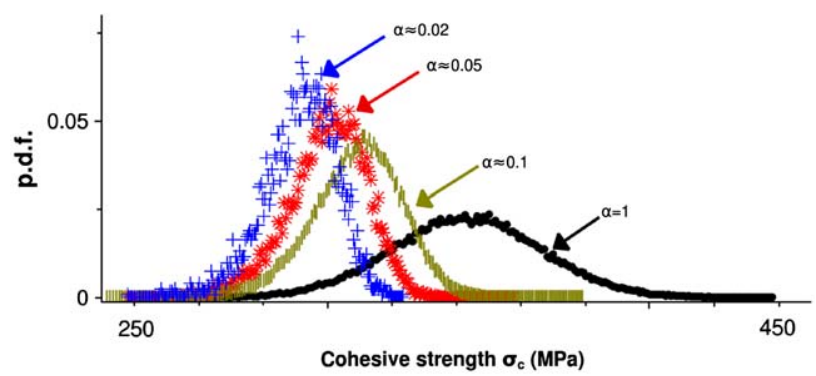

Fig. 14. Influence of $\alpha=N_{\text {frag }} / N_{\text {def }}$ on the broken p.d.f., for an initial Gaussian p.d.f.

value of $\alpha$ with the p.d.f. finit (Eq. (22)). For instance, a uniform p.d.f. $f^{\text {init }}$ theoretically coincides with the bisector function (Fig. 13).

From a mathematical point of view, monotonically decreasing p.d.f. can lead to convex curve. However, such p.d.f. seem to have no physical meaning: a monotonically decreasing p.d.f. $f^{\text {init }}$ refers to a material which has more weakest links than strong ones. Since brittle materials usually exhibit more macroscopic than microscopic defects, we have limited our study to p.d.f. leading to concave curves, as shown in Fig. 13.

Intermediate behaviors are situated between these two extreme cases. In order to characterize the function $\Psi$, several tests are carried out for the same initial distribution $f^{\text {init }}$ and several values of $\alpha$. In Fig. 14, the initial distribution is Gaussian with mean $\mu^{\text {init }}=345 \mathrm{MPa}$ and standard deviation $v^{\text {init }}=23.2 \mathrm{MPa}$ (Gauss 2 in Table 1 ). $\alpha=1$ represents the initial distribution $f^{\text {init }}$, and the three other values $(\alpha=0.1,0.05,0.02)$ result from a change in the strain rate and hence, in the number of fragments $N_{\text {frag }}$.

Fig. 15 plots the functions $\Psi$ associated to the four distributions Uniform 1, Weibull 2-1, Gauss 2 (Table 1) and $\beta=0.4$ (Eq. (24)). Each numerical test results in one point, the dotted lines are the fitting functions. Power laws appear to be adequate approximations and lead to

$$
\forall f^{\text {init }}, \exists a \in[0,1], \forall \alpha \in[0,1], \quad \Psi(\alpha)=\alpha^{a}
$$




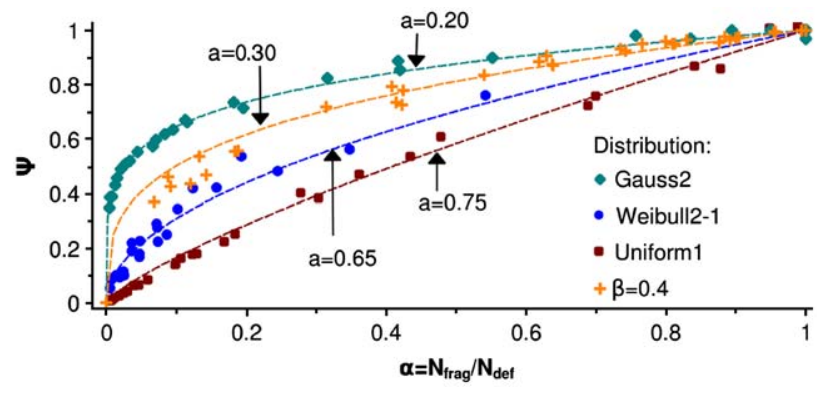

Fig. 15. Numerical evaluation of the communication parameter $a$ for four distinct initial p.d.f.

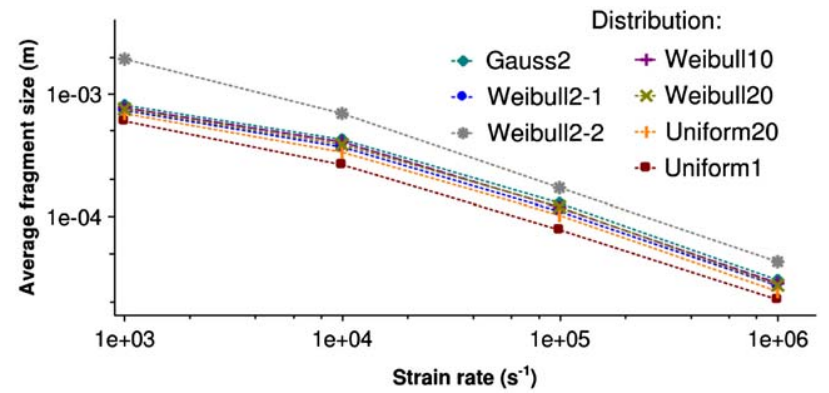

Fig. 16. Dispersion in the average fragment size when the strain rate and the microstructure vary.

where

$$
a=\left\{\begin{array}{lll}
0.20 & \text { for } \text { Gauss2 } \\
0.30 & \text { for } & \beta=0.4 \\
0.65 & \text { for } \text { Weibull2-1 } \\
0.75 & \text { for } \text { Uniform } 1
\end{array}\right.
$$

The distribution Gauss 2 leads thus to a more communicative process than the Weibull 2-1 and the Uniform 1 distributions. Since the values of the Gauss 2 weakest links vary regularly, their activation is not instantaneous, the stress waves have time to propagate and influence the neighboring defects. The slope of the p.d.f. $f^{\text {init }}$ appears to be essential in the communication process. This is confirmed by the $\beta=0.4$ distribution (Eq. (24)) which slope at $\sigma_{c, m i n}$ is comprised between the Gauss 2's and the Weibull 2-1's slopes.

In conclusion, the p.d.f. strongly influences the fragmentation process. The slope at $\sigma_{c, \min }$ plays a prevailing role in the activation of weak cohesive elements and a fortiori in the communication process. It can be quantified by the communication parameter $a$ which low values represent highly interactive processes.

\section{Scaling of the average fragment size}

In this section, we focus on heterogeneous materials with a high density of defects, such that the number of defects and the number of fragments are independent (plateau region described in Section 3.2). We look at the influence of the distribution of defects and the strain rate on the average fragment size. Fig. 16 plots the average fragment size for different initial distributions and for several strain rates. The scattering of the points underlines that defects play a prevailing role in the fragmentation process.

The idea of normalizing the axes to gather all these points into a single curve arises. In the normalization proposed by Zhou et al. for homogeneous materials, the normalization parameters were expressed in terms of $\sigma_{c}$ and $G_{c}$. Now, since the material is heterogeneous, $\sigma_{c}$ and $G_{c}$ are defined through their distributions instead of being a single value. The homogeneous normalization is not adequate anymore. With the aim at understanding separately the effect of the initial p.d.f. and the communication parameter $a$, we propose two normalizations. The first one only depends on the initial p.d.f. and not on the communication, while the second accounts for both. Thus, we define new semi-empirical normalization parameters:

$$
\begin{gathered}
\dot{\varepsilon}_{0, \text { new }}=\frac{\mu_{\text {init }}}{E t_{0}} \\
s_{0, \text { new }}=c_{\text {eff }} t_{0}
\end{gathered}
$$




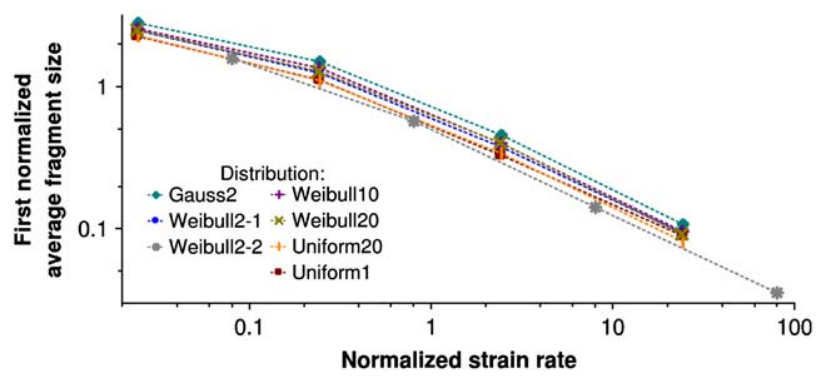

Fig. 17. Effect of the first normalization which only includes the minimum cohesive strength $\sigma_{c, \min }$ and the average of the initial distribution of defects $\mu_{\text {init. }}$.

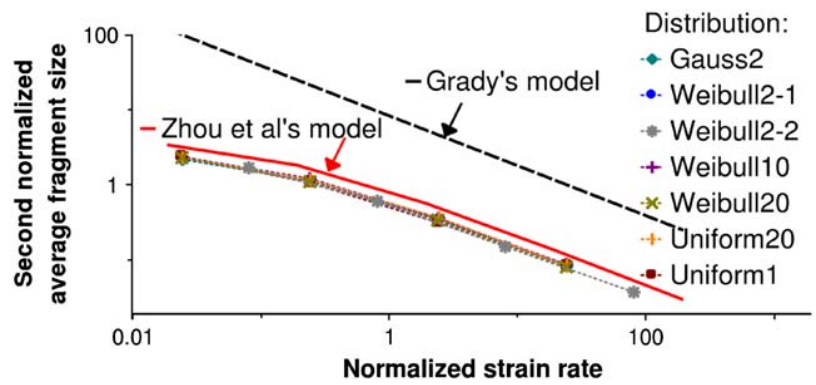

Fig. 18. Effect of the second normalization which includes $\sigma_{c, \text { min }}, \mu_{\text {init }}$ and the communication parameter $a$, and comparison to Grady's and Zhou et al.'s laws.

where

$$
c_{\text {eff }}= \begin{cases}c\left(\frac{\sigma_{c, \text { min }}}{\mu_{\text {init }}}\right)^{1 / 5} & \text { first normalization } \\ c \sqrt{\frac{2}{a+1}}\left(\frac{\sigma_{c, \text { min }}}{\mu_{\text {init }}}\right)^{1 / 5} & \text { second normalization }\end{cases}
$$

$\mu_{\text {init }}$ is the average of the initial distribution of defects, $\sigma_{c, \min }$ is the cohesive strength associated to the weakest link, $t_{0}$ is the characteristic opening time of the cohesive elements, and $a$ is the communication factor defined in Section 4.

Multiplying the wave speed $c$ by a function depending on the communication factor $a$ is necessary to include the influence of the interactions between cracks, on the average fragment size. For a homogeneous material, the p.d.f. tends to be a Dirac function, for which the function $\Psi$ is not defined anymore. If one considers that the Dirac function is a uniform distribution with a zero standard deviation, and that communications between cracks do not have time to establish, then $a \rightarrow 1$. It directly leads to $c_{e f f} \rightarrow c$. When the material is heterogeneous, the communication time affects the number of fragments. A low value of $a$ is associated to a process during which cracks strongly interact. Stress waves propagate fast compared to the crack opening time $t_{0}$, which justifies that the effective wave speed is a decreasing function of $a$. Note that the exponent $\frac{1}{5}$ in Eq. (28) was obtained to fit best our results.

In Figs. 17 and 18, the $x$-axis and $y$-axis are, respectively, $\overline{\dot{\varepsilon}}_{\text {new }}=\dot{\varepsilon} / \dot{\varepsilon}_{0, \text { new }}$ and $\bar{s}_{\text {new }}=s_{\text {average }} / s_{0, \text { new }}$. The effect of the first normalization is illustrated in Fig. 17. It brings the curves into three distinct groups: the Gaussian, the Weibull, and the Uniform. The shape of the p.d.f. appears to affect the gathering and needs thus to be included. Therefore, we propose to use the communication parameter a defined in Section 4, which is directly linked to this shape and to the rate of insertion of cohesive elements. Fig. 18 compares the final normalization to Grady's and Zhou et al.'s laws for homogeneous materials. The log-log plot underscores that our results exhibit a characteristic exponent which is very closed to Grady's minus twothirds exponent, and that we predict approximately the same non-dimensional number of fragments that Zhou et al. obtained for homogeneous materials.

Although empirical, our second normalization successfully merges the initially scattered points into a single curve. Following the shape of the function proposed by Zhou et al. (Section 2.5), the present curve can be fitted by

$$
\bar{s}_{\text {new }}=\frac{3}{1+4.5\left(\overline{\dot{\varepsilon}}_{\text {new }}\right)^{2 / 3}}
$$


Its developed form is

$$
s=t_{0} c_{e f f} \frac{3}{1+4.5\left(\frac{E t_{0}}{\mu_{\text {init }}}\right)^{2 / 3} \dot{\varepsilon}^{2 / 3}}
$$

where

$$
c_{\text {eff }}=c\left(\frac{2}{a+1}\right)^{1 / 2}\left(\frac{\sigma_{c, \min }}{\mu_{\text {init }}}\right)^{1 / 5}
$$

The parameters used in the simulations are:

- Young's modulus $E=275 \mathrm{GPa}$.

- Wave speed $c=10^{4} \mathrm{~m} \mathrm{~s}^{-1}$.

- Reference opening time $t_{0}=300 \mu \mathrm{s}$

along with defect distribution dependent parameters:

- Average of the initial distribution of defects $\mu_{\text {init }}$.

- Weakest link $\sigma_{c, \min }$.

- Communication parameter:

$$
a= \begin{cases}0.20 & \text { for Gaussian distribution } \\ 0.65 & \text { for Weibull distribution } \\ 0.75 & \text { for Uniform distribution }\end{cases}
$$

The resulting effective wave speeds are

$$
c_{\text {eff }}=c \begin{cases}1.21 & \text { for Gauss2 } \\ 1.07 & \text { for Weibull2-1 } \\ 0.94 & \text { for Weibull2-2 } \\ 1.08 & \text { for Weibull10 } \\ 1.09 & \text { for Weibull20 } \\ 0.84 & \text { for Uniform } 1 \\ 1.06 & \text { for Uniform20 }\end{cases}
$$

The proposed scaling law matches the initially scattered points, but highlights differences to prior analytical and numerical models. For rather defect-free materials (such as Uniform 20 in Table 1), we predict slightly more fragments than the one-dimensional numerical model of Zhou et al. (e.g. our average fragment size is about $22 \%$ smaller). This seems to be due to the specific implementation of the two-dimensional contact algorithm. Our computed average fragment size is also smaller by a factor of 10 than Grady's energy predictions which assumes that the process is energy-equilibrated. While valid for ductile materials, this assumption appears incomplete for the presently tested brittle solids. Indeed, in his first papers (Grady, 1982, 1988), Grady considers that the local kinetic and the elastic strain energies are converted into fracture energy at the onset of failure. In more recent work, Grady (2008) underlines that brittle solids undergo a non-equilibrated fragmentation. Indeed, since the onset of the fragmentation process is a nearly instantaneous, the damaged and the nondamaged regions barely communicate; at first, damage stays local. Hence, the non-damaged parts of the structure continue accumulating elastic strain energy, which is eventually converted into fracture energy, generating more fragments. Our simulations confirm Grady's (2008) second hypothesis. We have quantified that at high strain rates, most fragments result from the conversion of the potential energy accumulated in the damaged structure. In other words, kinetic energy is being converted into strain energy for a "long" period after peak strength. Since Grady's initial energy equilibrium law does not include this excess of strain energy, it predicts less fragments than our explicit numerical calculations.

Despite these dissimilarities, both the reference models and the present fitting curve exhibit a similar trend. Dynamic fragmentation tends asymptotically to a power-law with exponent equal to the inverse two-thirds. Ultimately, this suggests that the energetic criterion governs the dynamic regime. Indeed, statistical predictions would have inferred a dependence of the power exponent to the initial distribution of defects (Hild et al., 2003), and an impulse criterion would have lead to an inverse one-third exponent (Grady, 2006; Tuler and Butcher, 1968). Our results confirm that, even though dynamic brittle fragmentation is not energy-equilibrated, its asymptotic behavior is energy controlled.

\section{Conclusion}

In this paper, we have modeled the fragmentation of a heterogeneous brittle ring submitted to radial expansion. Numerical simulations underscore the prevailing role of defects in the fragmentation physical process and in the resulting 
fragment sizes. They also emphasize on the importance of accounting for stress release waves, which propagate away from crack initiation sites and may prevent damage nucleation at other defects.

We have quantified the crack initiation process by considering the degree of interactions between defects. It has lead us to define a new communication parameter which depends on the left tail of the initial probability density function of defects, and which accounts for the rate of insertion of cohesive elements. This communication parameter, combined with the wave speed, has been used to construct a normalization of the average fragment size as a function of strain rate. This normalization successfully unifies the initially scattered fragment size data into a unique curve. It efficiently scales the behavior of homogeneous and heterogeneous materials and highlights that, at high strain rates, dynamic fragmentation is characterized by a universal asymptotic behavior. The power-law with exponent minus two-thirds, predicted by Grady's energy-equilibrated theory, adequately fits our results. This indicates that the asymptotic limit of dynamic fragmentation is dominated by an energy criterion.

\section{Acknowledgments}

The authors gratefully acknowledge the support from ARO through the Grant no. W911NF-08-1-0150, and ARL through University of Nebraska Lincoln (Grant no. W911NF-04-2-0011).

\section{References}

Camacho, G.T., Ortiz, M., 1996. Computational modelling of impact damage in brittle materials. International Journal of Solids and Structures 33 (20-22), 2899-2938.

Drugan, W.J., 2001. Dynamic fragmentation of brittle materials: analytical mechanics-based models. Journal of the Mechanics and Physics of Solids 49 (6), 1181-1208.

Englman, R., Rivier, N., Jaeger, Z., 1987. Fragment-Size distribution in disintegration by maximum-entropy formalism. Philosophical Magazine B-Physics of Condensed Matter Statistical Mechanics Electronic Optical and Magnetic Properties 56, 751-769.

Espinosa, H.D., Zavatierri, P.D., Dwivedi, S.K., 1998. A finite deformation continuum discrete model for the description of fragmentation and damage in brittle materials. Journal of the Mechanics and Physics of Solids 46, 1909-1942.

Glenn, L.A., Chudnovsky, A., 1986. Strain-energy effects on dynamic fragmentation. Journal of Applied Physics 59 (4), $1379-1380$.

Grady, D.E., 1982. Local inertial effects in dynamic fragmentation. Journal of Applied Physics 53 (1), $322-325$.

Grady, D.E., 1988. The spall strength of condensed matter. Journal of the Mechanics and Physics of Solids 36 (3), $353-384$.

Grady, D.E., 2006. Comparison of hypervelocity fragmentation and spall experiments with tuler-butcher spall and fragment size criteria. International Journal of Impact Engineering 33, 305-315.

Grady, D.E., 2008. Fragment size distributions from the dynamic fragmentation of brittle solids. International Journal of Impact Engineering 35, $1557-1562$.

Grady, D.E., 2009. Dynamic fragmentation of solids. Shock Wave Science and Technology Reference Library, vol. 3. Springer, Berlin, Heidelberg (Chapter).

Hild, F., Denoual, C., Forquin, P., Brajer, X., 2003. On the probabilistic-deterministic transition involved in a fragmentation process of brittle materials. Computers and Structures 81 (12), 1241-1253.

Holian, B.L., Grady, D.E., 1988. Fragmentation by molecular dynamics: the microscopic “big bang”. Physical Review Letters 60 (14), $1355-1358$.

Hughes, T., 2000. The Finite Element Method: Linear Static and Dynamic Finite Element Analysis. Dover Publications, New York.

Kraft, R.H., Molinari, J.F., Ramesh, K.T., Warner, D.H., 2008. Computational micromechanics of dynamic compressive loading of a brittle polycrystalline material using a distribution of grain boundary properties. Journal of the Mechanics and Physics of Solids 56 (8), $2618-2641$.

Leadbetter, M.R., Lindgren, G., Rootzén, H., 1983. Extremes and Related Properties of Random Sequences and Processes. Springer Series in Statistics. Springer, Berlin.

Maiti, S., Rangaswamy, K., Geubelle, P.H., 2005. Mesoscale analysis of dynamic fragmentation of ceramics under tension. Acta Materialia 53 (3), 823-834.

Mott, N.F., 1943. Fragmentation of h.e. shells: a theoretical formula for the distribution of weights of fragments. United Kingdom Ministry of Supply, AC3642, March.

Mott, N.F., Linfoot, E.H., 1943. Ministry of Supply. AC 3348, January.

Paliwal, B., Ramesh, K.T., 2008. An interacting micro-crack damage model for failure of brittle materials disorder effects in dynamic fragmentation of brittle materials. Journal of the Mechanics and Physics of Solids 56, 896-923.

Raghupathy, R., Gazonas, G.A., Molinari, J.F., Zhou, F., 2006. Numerical convergence of the cohesive element approach in dynamic fragmentation simulations. In: Shock Compression of Condensed Matter, American Institute of Physics Conference Series, vol. 845, July, pp. 654-657.

Rosin, P., Rammler, E., 1933. The laws governing the fineness of powdered coal. Journal of the Institute of Fuel 7, 29-36.

Ryan, E.V., 2000. Asteroid fragmentation and evolution of asteroids. Annual Review of Earth and Planetary Sciences 28, $367-389$.

Shenoy, V.B., Kim, K.S., 2003. Disorder effects in dynamic fragmentation of brittle materials. Journal of the Mechanics and Physics of Solids 51, 2023-2035.

Tuler, F.R., Butcher, B.M., 1968. A criterion for the time dependence of dynamic fracture. International Journal of Fracture 4 (4), $431-437$.

Weibull, W., 1939. A statistical theory of strength of materials. In: Proceedings of the Ingeniors Vetenskapsakad, p. 151.

Wittel, F.K., Kun, F., Herrmann, H.J., Kroplin, B.H., 2005. Breakup of shells under explosion and impact. Physical Review E 71 (1), 016108.

Zhou, F., Molinari, J.F., Ramesh, K.T., 2005. A cohesive model based fragmentation analysis: effects of strain rate and initial defects distribution. International Journal of Solids and Structures 42, 5181-5207.

Zhou, F., Molinari, J.F., Ramesh, K.T., 2006a. Analysis of the brittle fragmentation of an expanding ring. Computational Materials Science 37 (1-2), 74-85.

Zhou, F., Molinari, J.F., Ramesh, K.T., 2006b. Analysis of the brittle fragmentation of an expanding ring. Computational Materials Science 37 (1-2), 74-85.

Zhou, F., Molinari, J.F., Ramesh, K.T., 2006c. Effects of material properties on the fragmentation of brittle materials. International Journal of Fracture 139 (2), 169-196. 